\title{
Liposomes prolong the therapeutic effect of anti-asthmatic medication via pulmonary delivery
}

This article was published in the following Dove Press journal:

International Journal of Nanomedicine

27 February 2012

Number of times this article has been viewed

\author{
Xiaoyu Chen \\ Wenhua Huang \\ Blenda Chi Kwan Wong \\ Linlin Yin \\ Yuen Fan Wong \\ Min Xu \\ Zhijun Yang
}

School of Chinese Medicine, Hong Kong Baptist University, Kowloon

Tong, Hong Kong
Correspondence: Zhijun Yang School of Chinese Medicine, Hong Kong Baptist University, 7 Baptist University Road, Kowloon Tong, Hong Kong

Tel +85 234 I | 296 I

Fax +85 234| |246|

Email yzhijun@hkbu.edu.hk
Purpose: The main objective of this study was to develop a novel aerosolized liposome formulation for pulmonary delivery of anti-asthmatic medication and to explore the relationship between the bioavailability and anti-asthmatic efficacy of such a formulation. Asthma treatment usually requires frequent administration of medication for sustained bronchodilating response. Liposomes are known for their capability for sustained drug release and thus would be a suitable delivery system for anti-asthmatic medication for prolonged therapeutic effect. Salbutamol sulfate (SBS) was chosen as the model drug in this study because of its high water solubility and fast absorption after administration.

Methods: SBS was efficiently encapsulated into liposomes by the vesicular phospholipid gel technique. SBS permeability across the pulmonary membrane of an Asian toad was determined by in vitro study. Intratracheal administration of liposomes labeled with the fluorescent dye $1,1^{\prime}$-dioctadecyltetramethyl indotricarbocyanine iodide (DiR) in a rat model was assessed by a small animal imaging system and pharmacokinetic analysis. Pharmacodynamic analysis was performed in guinea pigs using the Konzett-Rössler method.

Results: SBS was efficiently encapsulated into liposomes with encapsulation efficiency as high as $70 \%$. The particle size of the SBS liposome suspension was approximately $57 \pm 21 \mathrm{~nm}$. In the in vitro study of permeability across the pulmonary membrane of Asian toads, SBS from liposomes demonstrated a slower transport rate compared to free SBS solution. Pulmonary delivery of liposomes in a rat model showed that the liposomes were effectively distributed in the respiratory tract and lungs, and that the release of SBS from liposomes was sustained for at least 48 hours. Pharmacodynamic analysis in a guinea pig model showed that the anti-asthmatic effect of SBS liposomes persisted for up to 18 hours, whereas that of free SBS solution was less than 8 hours.

Conclusion: The overall results demonstrated that liposomes could increase the concentration and retention time of SBS in the lungs and therefore prolong its therapeutic effect.

Keywords: salbutamol sulfate, asthma, intratracheal administration, sustained release

\section{Introduction}

Asthma is a chronic pulmonary disease that has recently been rising in incidence in adults. ${ }^{1}$ The first selective $\beta_{2}$ receptor agonist salbutamol sulfate (SBS) has been widely used for the treatment of bronchospasm in conditions such as exercise-induced asthma ${ }^{2}$ and chronic obstructive pulmonary disease. ${ }^{3}$ Commercial pharmaceutical formulations of SBS include aerosols, injection fluids, mixtures, and tablets. ${ }^{4}$ While effective, oral or intravenous administration of SBS in high doses is often associated with adverse effects, including maternal and fetal tachycardia, maternal hyperglycemia, dyspnea, palpitations, anxiety, and oliguria. ${ }^{5}$ Moreover, SBS is subject to first-pass elimination 
in the liver when it is taken orally. ${ }^{6}$ As a result, SBS is now rarely delivered via the oral route and is usually given by inhalation. Pulmonary delivery stands out among alternative administration routes because of the direct targeting of drugs to the lungs, which reduces unwanted systemic activity or toxicity. Conventional SBS aerosol formulations demonstrate a fast pharmacokinetic profile and thus the dosage must be repeated every 4-6 hours to sustain a bronchodilating response. ${ }^{7}$ Accordingly, a novel SBS dosage form with a long-lasting effect is desperately needed to treat asthma.

To convey a sufficient dose of drug to the lungs, suitable drug carriers are required. These can be either liquid, ${ }^{8-14}$ solid, ${ }^{14,15}$ or gaseous formulations. Over the years, liposomes have received widespread attention as a pulmonary drug delivery system, ${ }^{8,16}$ providing an attractive means for noninvasive delivery and sustained release of drugs for treating chronic diseases, such as asthma and diabetes. Liposomemediated pulmonary drug delivery may increase drug retention time in the lungs while reducing extrapulmonary side effects, resulting in enhanced therapeutic efficacy. ${ }^{17}$

The use of liposomes for topical administration may retain and localize SBS within a reservoir at the site of administration. Thus, liposome formulations with high SBS carrying capacities and improved retention times are desired. In this study, an aerosolized liposome carrier for pulmonary delivery of SBS was developed. The drug was encapsulated into liposomes by the vesicular phospholipid gel (VPG) technique. The encapsulation efficiency (EE) and particle size of liposomes were optimized. The in vitro permeability of SBS liposome formulation across Asian toad pulmonary membrane was compared to that of free SBS solution. The distribution and elimination of liposomal SBS in the lungs and its pharmacokinetic and pharmacodynamic parameters were studied in animal models via direct pulmonary administration.

\section{Materials and methods Materials}

SBS was purchased from Changzhou Yabang Pharmaceutical Co, Ltd (Changzhou, China). Soybean phosphatidylcholine (SPC) was purchased from Taiwei Pharmaceutical Co, Ltd (Shanghai, China). The isotonic agents used in this study were glucose (Riedelde Haën, Seelze, Germany), $\alpha$-lactose monohydrate (Sigma Aldrich, St Louis, MO), mannitol (International Laboratory, San Bruno, CA), and trehalose (International Laboratory). Acetonitrile (HPLC grade) was supplied by Labscan Asia Co, Ltd (Bangkok, Thailand). Deionized water was generated by a Milli Q ultrapure water system (Millipore, Billerica, MA). Ethanol (Labscan Asia Co, Ltd) was of analytical grade. 1,1'-dioctadecyltetramethyl indotricarbocyanine iodide (XenoLight DiR) was obtained from Caliper LifeSciences (Hopkinton, MA). Histamine was purchased from Sigma Aldrich.

\section{Animals}

Male Sprague Dawley rats with an average weight of $220 \pm 20 \mathrm{~g}$ and male guinea pigs with an average weight of $480 \pm 30 \mathrm{~g}$ were used (Laboratory Animal Services Center, The Chinese University of Hong Kong). All procedures involving animals were carried out according to the guidelines of the Committee on the Use of Human and Animal Subjects in Teaching and Research of Hong Kong Baptist University and the Health Department of Hong Kong Special Administrative Region.

\section{Methods}

\section{Preparation and dilution of SBSVPG and SBS DiR VPG}

The preparation of VPG was conducted based on the protocol described by Brandl and Massing. ${ }^{18}$ For production of SBS VPG (40\% of SPC and $8.3 \%$ of SBS by mass), SPC was dispersed in $10 \mathrm{~mL}$ of aqueous SBS solution (13.9\% of SBS by mass) by manual shaking in a stoppered tube for over 2 minutes. After incubation in a water bath at $60^{\circ} \mathrm{C}$ for 2 hours, the mixture was stirred using a homogenizer (Ultra Turrax T18; IKA, Staufen, Germany) for 5 minutes to form a semisolid homogenous product. SBS-VPG was formed by incubation in a water bath at $60^{\circ} \mathrm{C}$ for 12 hours and was stored at $4{ }^{\circ} \mathrm{C}$ before use. SBS liposome suspension $(4 \mathrm{mg} / \mathrm{mL})$ was prepared by diluting $0.48 \mathrm{~g}$ of SBS VPG with $10 \mathrm{~mL}$ of $\alpha$-lactose solution (77 $\mathrm{mg} / \mathrm{mL}$ ). The same procedures were applied for the production of SBS DiR VPG (40\% of SPC and $8.3 \%$ of SBS by mass), except that SPC was dispersed in aqueous SBS solution containing $200 \mu \mathrm{L}$ of DiR ethanol solution (8.33 $\mathrm{mg} \mathrm{DiR} / \mathrm{mL}$ ) and then SBS DiR VPG was stored and protected from light at $-20^{\circ} \mathrm{C}$ before use.

\section{Characterization of liposome SBS}

The particle size distribution of the liposomes was determined by using the DELSA 440SX Zeta Potential Analyzer (Beckman Coulter, Brea, CA). Measurement was made at $25^{\circ} \mathrm{C}$.

The EE of SBS in the liposomes was determined by the Microcon method. ${ }^{19,20}$ Briefly, an aliquot of the liposome suspension $(200 \mu \mathrm{L})$ was added to the sample reservoir of a Microcon centrifugal filter device containing an ultrafiltration membrane (NMWL 10000, Millipore) and centrifuged 
(Avanti $^{\circledR} \mathrm{J}-\mathrm{E}$, Beckman Coulter) at $17400 \mathrm{~g}$ for 15 minutes at $4^{\circ} \mathrm{C}$. $50 \mu \mathrm{L}$ of filtrate was diluted with $1450 \mu \mathrm{L}$ of $80 \%$ ethanol and assayed to determine the concentration of the unencapsulated SBS by a UV spectrophotometer (Cary 50 Tablet; Varian Technologies Asia Ltd, Taipei, Taiwan) at $276 \mathrm{~nm}$. To determine the total SBS concentration, the liposome structure was destroyed by adding $1450 \mu \mathrm{L}$ of $80 \%$ ethanol to $50 \mu \mathrm{L}$ of the original liposome suspension. EE was calculated by the following formula:

$\mathrm{EE}=[($ Total drug - Unencapsulated drug $) /$ Total drug $] \times 100 \%$ $=\left(\mathrm{C}_{\mathrm{s}} \mathrm{V}_{\mathrm{s}}-\mathrm{C}_{\mathrm{f}} \mathrm{V}_{\mathrm{f}}\right) / \mathrm{C}_{\mathrm{s}} \mathrm{V}_{\mathrm{s}} \times 100 \% \approx\left(\mathrm{C}_{\mathrm{s}}-\mathrm{C}_{\mathrm{f}}\right) / \mathrm{C}_{\mathrm{s}} \times 100 \%$

in which $\mathrm{C}_{\mathrm{s}}$ and $\mathrm{C}_{\mathrm{f}}$ are the SBS concentrations in the original liposome suspension and filtrate, respectively, whereas $\mathrm{V}_{\mathrm{s}}$ and $\mathrm{V}_{\mathrm{f}}$ are the volumes of the original liposome suspension and filtrate, respectively. $\mathrm{V}_{\mathrm{f}}$ can be approximated to $\mathrm{V}_{\mathrm{s}}$ because $\mathrm{V}_{\mathrm{f}}=\mathrm{V}_{\mathrm{s}}-\mathrm{V}_{1}$ where $\mathrm{V}_{1}$ represents the volume of the liposome vesicles and $\mathrm{V}_{1}$ was less than $5 \%$ of $\mathrm{V}_{\mathrm{s}}$.

\section{Transport of SBS across pulmonary membrane}

SBS permeability across the pulmonary membrane of an Asian toad (an amphibian similar to Xenopus) was determined according to the method previously reported by other groups. ${ }^{21-23}$ The toad was anesthetized by ether and then underwent ventral incision to expose the thoracic cavity. The lungs were excised by severing the tracheoglottis and washed with Ringer's solution $(110 \mathrm{mmol} / \mathrm{L} \mathrm{NaCl}, 2.4 \mathrm{mmol} / \mathrm{L}$ $\mathrm{NaHCO}_{3}, 2.4 \mathrm{mmol} / \mathrm{L} \mathrm{KCl}, 1 \mathrm{mmol} / \mathrm{L} \mathrm{CaCl}_{2}, 5.5 \mathrm{mmol} / \mathrm{L}$ glucose, $\mathrm{pH}$ 7.4). After that, the lungs were cut open along the pulmonary artery to obtain a sheet membrane, both sides of which were gently washed with Ringer's solution. The sheet membrane was mounted onto a horizontal diffusion cell (PermeGear, Inc, Hellertown, PA). The volume of both the donor chamber and acceptor chamber was $6 \mathrm{~mL}$. The temperature of the chambers was maintained at $37^{\circ} \mathrm{C}$. SBS liposome suspension ( $3 \mathrm{~mL}$ ) or free SBS solution $(3 \mathrm{~mL})$ of the same concentration was mixed with $3 \mathrm{~mL}$ of Ringer's solution and added to the donor chamber. Another $6 \mathrm{~mL}$ of Ringer's solution was directly added to the acceptor chamber. The solutions in both chambers were stirred with a magnetic stirrer to maintain homogeneity. To determine the initial SBS concentration, $50 \mu \mathrm{L}$ was withdrawn from the donor chamber immediately after SBS liposome suspension or free SBS solution was added; the SBS solution aliquot was diluted 100 -fold with deionized water for further assay, whereas the SBS liposome aliquot was first diluted five-fold with $80 \%$ ethanol to destroy the liposome structure and then further diluted 20-fold with deionized water. Afterwards, $1 \mathrm{~mL}$ of solution was withdrawn once every 15 minutes from the acceptor chamber for a period of 4 hours, and an equal volume of fresh Ringer's solution was added to refill the chamber each time. The electrical resistance of the pulmonary membrane was monitored (EVOM System; World Precision Instruments, Inc, Sarasota, FL) to ensure its integrity during the experiment. Each aliquot of withdrawn solution was filtered with a $0.45 \mu \mathrm{m}$ membrane filter (Disposable Sterile Syringe Filters, Cellulose Acetate Membrane; Asahi Glass Co, Ltd, Tokyo, Japan), and then $400 \mu \mathrm{L}$ of the filtrate was injected into the HPLC (Agilent 1100, Santa Clara, CA) to determine the amount of SBS that had crossed the membrane at each time point. HPLC analysis was performed with the C18 column $(250 \times 4.60 \mathrm{~mm}$; Phenomenex, Torrance, CA) at $40^{\circ} \mathrm{C}$ and the UV detector at $276 \mathrm{~nm}$. The injection volume was $20 \mu \mathrm{L}$. The mobile phase was a mixture of acetonitrile and sodium dihydrogen phosphate buffer $(0.03 \mathrm{M})$ in a ratio of $10: 90(\mathrm{~V}: \mathrm{V})$ with the addition of $1 \mathrm{M}$ phosphoric acid solution to obtain a $\mathrm{pH}$ of 3.5 , and the flow rate was $1 \mathrm{~mL} / \mathrm{minute}$.

\section{In vivo animal studies}

In vivo fluorescence imaging of DiR liposome distribution in rat lungs

Twenty-seven male Sprague Dawley rats were fasted for 12 hours before the experiment. Rats were weighed and then anesthetized by intraperitoneal administration of $7 \%$ chloral hydrate at a dosage of $0.5 \mathrm{~mL}$ per $100 \mathrm{~g}$ of body weight. DiR SBS liposome suspension was administered directly via spray instillation to the lungs using a spray instillator (Model 1A-1B MicroSprayer ${ }^{\circledR}$; Penn Century, Wyndmoor, PA) at a dosage of $2 \mathrm{mg} \mathrm{SBS} / \mathrm{kg}$. At 15 minutes and 1, 2, 4, 8, 12, 18, 24 , and 48 hours ( $n=3$ for each time point) after drug administration, rats were sacrificed and the thoracic cavity was opened. The DiR SBS liposome distribution in rat lungs was assessed at each time point using a small animal imaging system (IVIS ${ }^{\circledR}$ Lumina XR; Caliper LifeSciences) with Living Image software (v 4.0; Caliper LifeSciences).

\section{Pharmacokinetic analysis in rat plasma}

Ten Sprague Dawley rats were randomly divided into two groups and fasted for 12 hours before the experiment. Rats were weighed and then anesthetized by intraperitoneal administration of $7 \%$ chloral hydrate at a dosage of $0.5 \mathrm{~mL}$ per $100 \mathrm{~g}$ of body weight. SBS liposome suspension or free SBS solution was delivered intratracheally using the spray instillator at a dosage of $2 \mathrm{mg} \mathrm{SBS} / \mathrm{kg}$ ( $\mathrm{n}=5$ per group). Blood samples 
$(0.4 \mathrm{~mL})$ were collected from the end of rat tails into, $1000 \mathrm{IU} / \mathrm{mL}$ heparinized centrifuge tubes at 5 minutes and $0.5,1,2,3,5,7,12,24$, and 48 hours after drug administration. Each sample was centrifuged at $1485 \mathrm{~g}$ for 10 minutes at $4^{\circ} \mathrm{C}$ to separate the plasma from the blood cells. One hundred microliters of plasma was mixed with $800 \mu \mathrm{L}$ of acetonitrile by vortex for 3 minutes. The denatured protein precipitate was then separated by centrifugation at $13,362 \mathrm{~g}$ for 10 minutes at $4^{\circ} \mathrm{C}$. The supernatant was transferred into a new centrifuge tube and the organic solvent was removed by vacuum at $25^{\circ} \mathrm{C}$ for 6 hours. The resulting residue was dissolved in $100 \mu \mathrm{L}$ of deionized water with $0.1 \%$ formic acid by vortex for 2 minutes. The solution was then centrifuged at $13,362 \mathrm{~g}$ for 10 minutes at $4^{\circ} \mathrm{C}$. Fifty microliters of the supernatant was withdrawn for SBS determination using UPLC MS/MS analysis.

\section{Concentration and disposition of SBS in rat lungs}

Fifty-four Sprague Dawley rats were randomly divided into two groups and fasted for 12 hours before the experiment. Rats were weighed and then anesthetized by intraperitoneal administration of $7 \%$ chloral hydrate at a dosage of $0.5 \mathrm{~mL}$ per $100 \mathrm{~g}$ of body weight. SBS liposome suspension or free SBS solution was delivered intratracheally using the spray instillator at a dosage of $2 \mathrm{mg} \mathrm{SBS} / \mathrm{kg}$. At 15 minutes and 1, 2, 4, 8, 12, 18, 24,48 hours after drug administration $(n=3$ for each time point), each animal was sacrificed for dissection of the lungs. Lung tissue samples were weighed and homogenized in $2 \mathrm{~mL}$ of physiological saline. The homogenate was then mixed with $12 \mathrm{~mL}$ of acetonitrile by vortex for 3 minutes. The denatured protein precipitate was then separated by centrifugation at $30,966 \mathrm{~g}$ for 15 minutes at $4{ }^{\circ} \mathrm{C}$. The supernatant was transferred into a new centrifuge tube and the organic solvent was removed by vacuum at $25^{\circ} \mathrm{C}$ for 12 hours. The resulting residue was dissolved in $3 \mathrm{~mL}$ of deionized water with $0.1 \%$ formic acid by vortex for 2 minutes. The solution was then filtered by a cellulose acetate membrane filter $(0.2 \mu \mathrm{m}$, Grace Davison Discovery Sciences, Augusta, GA). One milliter of the filtrate was withdrawn for SBS determination using UPLC-MS/MS analysis as stated below.

\section{Pharmacodynamic study of guinea pigs}

Pharmacodynamic analysis was performed in guinea pigs using the Konzett-Rössler method. ${ }^{24}$ The advantage of this method is that not only the bronchodilator activity but also the systemic influences, such as cardiovascular events, due to SBS can be monitored simultaneously in an animal model. ${ }^{25}$ Briefly, each guinea pig was put into a plastic box (4.5 L) and then $0.4 \%$ histamine saline solution was sprayed into the box for 20 seconds at the rate of $70 \mathrm{~L} /$ minute. Asthma latent period is defined as the time it takes for the animal to twitch and fall down after spraying of histamine. ${ }^{26}$ Guinea pigs with an asthma latent period exceeding 120 seconds were not included in this study.

Selected guinea pigs were randomly divided into three groups with six in each group. Each guinea pig was weighed and then anesthetized by intraperitoneal administration of $10 \%$ chloral hydrate at a dosage of $4 \mathrm{~mL}$ per $\mathrm{kg}$ of body weight. Each group of guinea pigs received SBS liposome suspension, free SBS solution, or saline blank solution through intratracheal delivery using the spray instillator at a dosage of $2 \mathrm{mg} \mathrm{SBS} / \mathrm{kg}$. At 2, 4, 6, 8, 12, 18, and 24 hours after drug administration, each animal was subject to the Konzett-Rössler experiment as described above. The asthma latent periods were recorded at each time point.

\section{UPLC-MS/MS analysis}

The analysis of SBS levels in vivo was carried out using UPLC (Acquity Ultra Performance Liquid Chromatography; Waters, Milford, MA) and MS/MS (MicroTOF-Q; Bruker Daltonics Inc, Billerica, MA), with the $\mathrm{C} 18$ column $(1.7 \mu \mathrm{m}$, $2.1 \times 100 \mathrm{~mm}$, Waters) at $40^{\circ} \mathrm{C}$. The mobile phase was $0.1 \%$ formic acid in deionized water, with a flow rate of $0.2 \mathrm{~mL} / \mathrm{minute}$. Ten microliters of the sample was used for the UPLC MS/MS analysis. SBS showed mass to electric charge ratio values of 148 and 166 in MS spectra.

\section{Data analysis}

The pharmacokinetic parameters were calculated and summarized by Thermo Kinetica (v 5.0; Cypress Software Inc, Langley, WA).

All data were analyzed using SPSS software (v 11.0; IBM Corp, Armonk, NY). Data were presented as mean \pm standard deviation (SD) and evaluated by Student's $t$-test to analyze the differences in pharmacokinetic parameters between two groups and by ANOVA test to analyze the differences in EE and asthma latent period when there were more than two groups; $P$ values below 0.05 indicate that the differences are statistically significant.

\section{Results and discussion} Preparation of SBS VPG and SBS DiR VPG VPG are semisolid matrices of densely packed liposomes, mainly consisting of small unilamellar vesicles, with high EE. ${ }^{27,28}$ VPG can be transformed into a "conventional" smallsized liposome dispersion by the addition of excess aqueous medium and gentle mechanical agitation shortly before use. 
During this dilution process, if appropriate conditions are maintained, the preformed vesicles in VPG would remain intact and retain their drug load. ${ }^{19}$ This has been confirmed by the present study using VPG to develop a liposomal carrier system for SBS. The particle sizes of SBS liposomes that were formed by dispersion of VPG were shown to range from 33 to $58 \mathrm{~nm}$ (Table 1). VPG-derived liposome formulations contain high lipid content contributing to a considerably increased ratio of aqueous volume inside the vesicles to the surrounding aqueous volume and thus are suitable for entrapping water-soluble substances with high $\mathrm{EE}(>50 \%) .{ }^{29} \mathrm{In}$ addition, use of formulations with high EE has the benefit that there is no need to remove the unencapsulated drug before use, making the production process more efficient. ${ }^{30-32}$ In order to investigate the effect of SPC content on the EE of VPG liposomes for SBS, a series of liposome dispersions were prepared with increasing SPC/VPG from 250 to $500 \mathrm{mg} / \mathrm{g}$. The EE was determined as the ratio of SBS concentration in the vesicle core to that in the total liposome suspension. As shown in Table 1, the EE was increased by increasing the SPC content in the original VPG $(P<0.05)$, suggesting that an increase of SPC/VPG (from 250 to $400 \mathrm{mg} / \mathrm{g}$ ) could lead to a higher volume of the vesicle core. However, it should also be noted that there was little increase in EE when SPC/VPG was above $400 \mathrm{mg} / \mathrm{g}$. Complete hydration of phosphatidylcholine would require a water-to-lipid ratio higher than 45:55. ${ }^{18}$ In this study's VPG formulation, the water-to-lipid ratio was 60:40 and 50:50 when SPC/VPG was 400 and $500 \mathrm{mg} / \mathrm{g}$, respectively. At $500 \mathrm{mg} / \mathrm{g}$, there was enough water present to completely hydrate the SPC lipid, but not enough to increase the aqueous core within the lipid vesicles. Thus, there was only a slight increase in the EE when SPC/VPG rose from 400 to $500 \mathrm{mg} / \mathrm{g}$. For the subsequent studies, an SPC concentration of $400 \mathrm{mg} / \mathrm{g}$ was used to prepare SBS liposomes.

Incorporation of DiR into liposomes was investigated in two separate experiments. DiR liposome suspension, which was prepared by hydrating the DiR VPG (SPC $400 \mathrm{mg} / \mathrm{g}$ ),

Table I Effect of SPC content in VPG on the liposome particle sizes and the EE of SBS in the liposome suspension (mean $\pm S D, n=3$ )

\begin{tabular}{lll}
\hline SPC/VPG (mg/g) & EE\% & Particle size $(\mathbf{n m})$ \\
\hline 250 & $35.12 \pm 0.99$ & $33 \pm 5.0$ \\
300 & $40.47 \pm 0.14$ & $47 \pm 10$ \\
400 & $57.11 \pm 0.11$ & $57 \pm 21$ \\
500 & $58.92 \pm 0.07$ & $58 \pm 26$ \\
\hline
\end{tabular}

Note: ${ }^{a} P<0.05$ for comparison between any two groups using ANOVA test. Abbreviations: EE, encapsulation efficiency; SBS, salbutamol sulfate; SD, standard deviation; SPC, soybean phosphatidylcholine; VPG, vesicular phospholipid gel. was added into the Microcon centrifugal filter device and centrifuged at $17,400 \mathrm{~g}$ for 15 minutes at $4^{\circ} \mathrm{C}$. Fifty microliters of filtrate was diluted with $1450 \mu \mathrm{L}$ of $80 \%$ ethanol and the unencapsulated DiR was analyzed by a fluorospectrophotometer (LS 55 Luminescence Spectrometer, PerkinElmer, Waltham, MA) at an excitation wavelength of $748 \mathrm{~nm}$ and an emission wavelength of $780 \mathrm{~nm}$. The filtrate showed no fluorescent intensity. In another experiment where liposome suspension was subject to extrusion through a 200nm polycarbonate membrane, there was no change of fluorescent intensity in the suspension before or after extrusion. These results indicated that DiR was not present in the aqueous phase of the suspension but mostly encapsulated into the lipid bilayer; this is in agreement with the lipophilic property of DiR. It has been reported that the two 18 carbon chains of DiR could insert into the liposome membrane, resulting in specific and stable liposome staining, with negligible dye transfer between liposomes and tissue cells. ${ }^{33}$

\section{Effect of isotonic agents on the EE of SBS in liposomes}

To further increase the EE of SBS in liposomes, the VPG with $400 \mathrm{mg} / \mathrm{g}$ SPC was hydrated with solutions of isotonic agents to prevent the release of SBS from the vesicles during conversion of the VPG into liposome suspension. The EE of SBS in liposomes reached $70.71 \% \pm 1.55 \%$ when hydrated with lactose solution. This EE value was found to be higher than that of the liposome suspension hydrated by deionized water $(57.11 \% \pm 0.11 \%)$ or by other isotonic solutions (Table 2, $P<0.05$ ). As the mass ratio of SBS (molar mass $=576.7 \mathrm{~g} / \mathrm{mol}$ ) in the VPG formulation was $8.3 \%$, the molar concentration of SBS in the aqueous phase of the VPG was calculated to be $0.28 \mathrm{M}$, and one molecule of SBS may dissociate into different ions in the aqueous solution. It can be inferred that the dispersion medium for VPG should be near isotonic to the aqueous phase within the vesicles. Isotonic agents could adjust the osmotic pressures in the inner and outer aqueous phases of vesicles to prevent the leakage of water-soluble SBS from the core of these vesicles when VPG is dispersed to form liposome suspension. The structure of lipid vesicles may also be changed by many other factors, such as the solubility of the isotonic agent and viscosity of the dispersion medium. Isotonic agents used in this study have different solubilities in water; the solubilities of glucose, mannitol, trehalose, and lactose at room temperature are 91, 22, 69, and $19 \mathrm{~g}$ in $100 \mathrm{~mL}$ of water, respectively. Other physical and chemical properties of isotonic agents may also affect their interaction with lipids and thus the EE of 
Table 2 Optimization of EE (mean \pm SD, $n=3$ ) of SBS in VPG liposomes by hydration with different concentrations of isotonic agents

\begin{tabular}{llll}
\hline Isotonic & \multicolumn{2}{l}{ Concentration } & EE (\%) \\
\cline { 2 - 3 } agent & $\mathbf{m g} / \mathbf{m L}$ & $\mathbf{M}$ & \\
\hline Glucose & 19.2 & 0.11 & $60.32 \pm 0.37$ \\
& 38.4 & 0.21 & $61.52 \pm 0.11$ \\
& 50.4 & 0.28 & $63.99 \pm 0.64$ \\
Mannitol & 76.8 & 0.43 & $65.75 \pm 0.46$ \\
& 115.2 & 0.64 & $66.29 \pm 1.57$ \\
& 19.2 & 0.11 & $65.27 \pm 0.60$ \\
& 38.4 & 0.21 & $62.53 \pm 0.81$ \\
Trehalose & 50.4 & 0.28 & $62.45 \pm 0.87$ \\
& 76.8 & 0.43 & $65.60 \pm 0.56$ \\
& 115.2 & 0.64 & $63.22 \pm 1.20$ \\
& 19.2 & 0.06 & $61.09 \pm 0.15$ \\
& 38.4 & 0.11 & $61.95 \pm 0.69$ \\
Lactose & 76.8 & 0.22 & $60.71 \pm 0.65$ \\
& 95.8 & 0.28 & $64.50 \pm 0.75$ \\
& 115.2 & 0.34 & $65.27 \pm 0.60$ \\
& 19.2 & 0.06 & $60.46 \pm 3.01$ \\
& 38.4 & 0.11 & $63.70 \pm 0.23$ \\
& 76.8 & 0.22 & $70.71 \pm 1.55^{a}$ \\
& 95.8 & 0.28 & $69.84 \pm 0.77$ \\
& 115.2 & 0.34 & $67.03 \pm 0.47$ \\
\hline Notes: & & &
\end{tabular}

Notes: The SBS concentration used for preparation of VPG liposomes was 83.4 $\mathrm{mg} / \mathrm{g}$. The average molar concentration of SBS in the aqueous phase of VPG was $0.28 \mathrm{M}$. a $P<0.05$ for comparison with other groups except for the molar concentration of lactose $0.28 \mathrm{M}$ using analysis of variance test.

Abbreviations: $\mathrm{EE}$, encapsulation efficiency; SBS, salbutamol sulfate; SD, standard deviation; VPG, vesicular phospholipid gel.

SBS in liposomes. This is reflected by the observation that the EE was not correlated with the concentration of isotonic agents. However, the study's data suggest that the osmotic pressure of the dispersion medium could be one important factor for maintaining high EE in VPG-derived liposome formulations. In this study, the EE of SBS in liposomes was optimized by adjusting the molar ratio of lactose closely to the average concentration of SBS in the inner aqueous phase of lipid vesicles. It was confirmed that the presence of DiR did not significantly change the EE of SBS in liposomes with lactose hydration $(\mathrm{EE}=68.22 \% \pm 1.78 \%)$.

\section{Effect of liposomes on the transport rate of SBS across the pulmonary membrane}

Figure 1 shows the transport profiles of SBS across the pulmonary membrane in vitro for comparison between the SBS liposome suspension and free SBS solution. SBS from liposome suspension displayed a relatively slower transport rate over the course of time when compared to free SBS solution. Interactions between liposomes and cells of the pulmonary membrane may

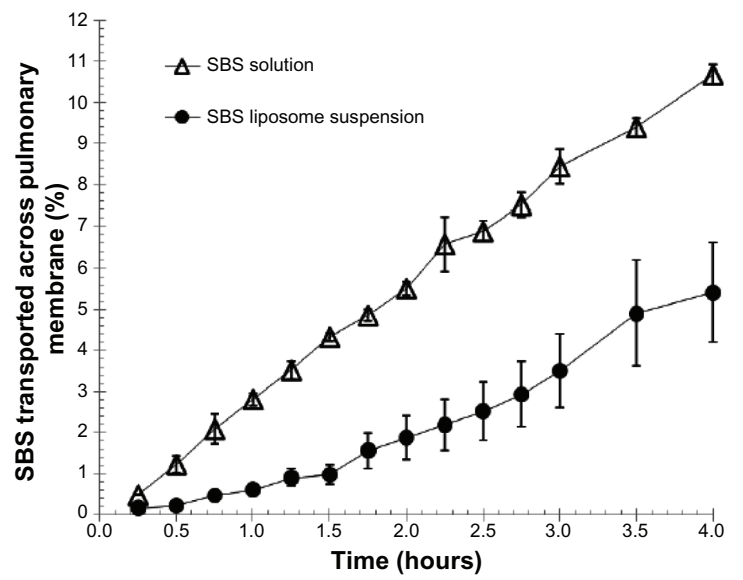

Figure I Effect of liposomes on the transport rate of SBS across Asian toad pulmonary membrane in vitro.

Notes: The concentrations of SBS in liposome suspension $(\bullet)$ and control solution $(\Delta)$ were $2.11 \mathrm{mg} / \mathrm{mL}$ and $2.22 \mathrm{mg} / \mathrm{mL}$, respectively. Data were expressed as mean $\pm S D(n=3)$.

Abbreviations: SBS, salbutamol sulfate; SD, standard deviation.

influence the release rate of SBS from liposomes. A previous report has described how some liposomes could be incorporated into the pulmonary membrane. ${ }^{34-36}$ Therefore, it is likely that there could be other forces besides diffusion that drive the transport of encapsulated SBS across the pulmonary membrane. Since the liposome vesicle was much larger than the SBS molecule in size, it was estimated that the transport rate of SBS from liposomes should be at least a dozen times slower than that of free SBS molecules. However, this study demonstrated only about a two-fold decrease in the transport rate of SBS from liposomes after 4 hours (Figure 1). This could be explained by the possibility that some liposomes were damaged, resulting in the release of entrapped SBS before interacting with cells of the pulmonary membrane. Nonetheless, the data suggest that liposomes could slow down the migration of SBS across the pulmonary membrane and thus increase the relative bioavailability of SBS.

\section{In vivo animal studies}

In vivo fluorescence imaging of DiR-liposome distribution in rat lungs

In studies involving pulmonary drug administration, it is important to confirm whether the dosage has been effectively administered into the lungs. Real time in vivo fluorescence imaging is a very effective and widely applicable technology for studying drug deposition in tissues. Temporal and spatial data can be collected by labeling drugs with a fluorescent molecule such as a dye or quantum dot prior to administration into an animal model. ${ }^{37}$ In the present study, DiR, a near 


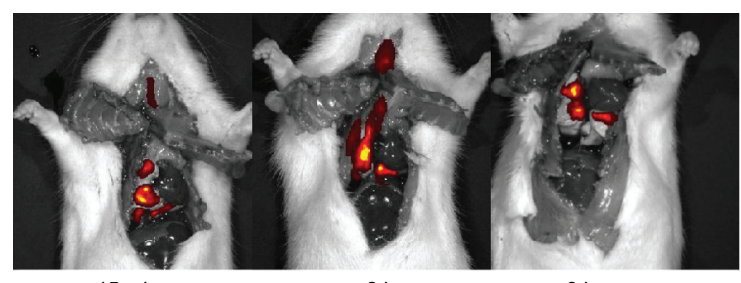

$15 \mathrm{~min}$

$2 \mathrm{~h}$

$8 \mathrm{~h}$

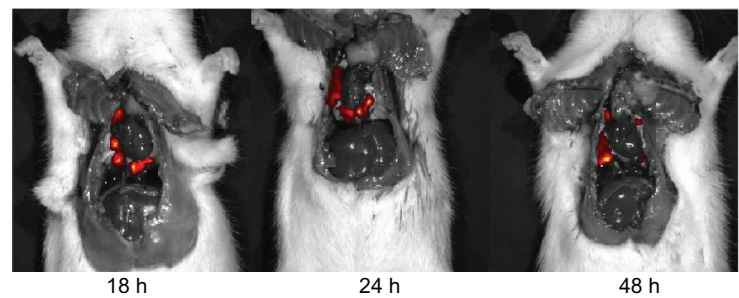

Figure 2A Fluorescence imaging of DiR SBS liposome disposition in rat lungs at different time points after intratracheal administration.

Abbreviations: SBS, salbutamol sulfate; DiR, I, I'-dioctadecyltetramethyl indotricarbocyanine lodide.

infrared fluorescent cyanine dye, was used for staining the lipid membrane. After intratracheal administration of DiR liposomes using the spray instillator, rats were sacrificed to expose the thoracic cavity for better fluorescence imaging effect. Serial images of the lungs showed persistent disposition of DiR SBS liposomes through the tracheobronchial tree (Figure 2A). Of important note, DiR SBS liposomes were observed in rat lungs even at 48 hours after drug administration, suggesting that liposomes could remain in the lungs and release SBS to exert sustained therapeutic effect. In addition, the elimination rate constant of DiR incorporated in the bilayer of the liposome membrane was calculated in order to estimate the elimination rate of liposomes in rat lungs. As shown in Figure 2B, the total radiation efficiency of DiR in

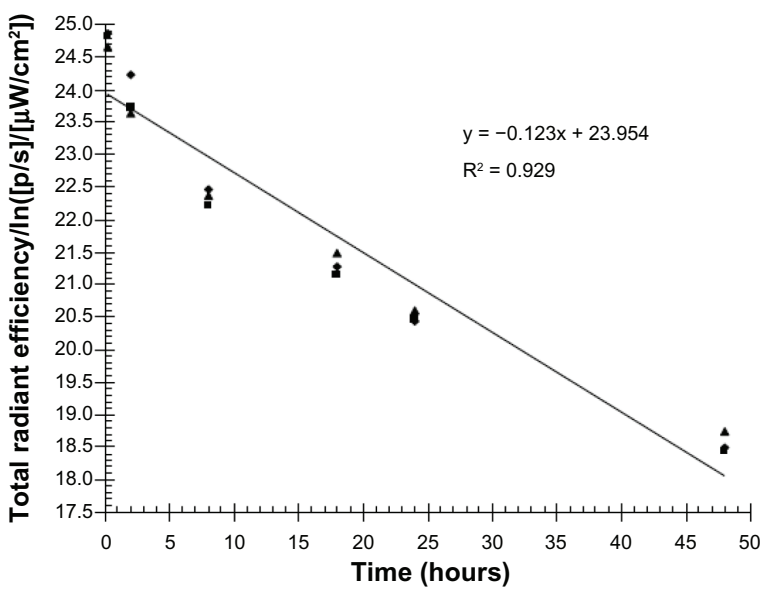

Figure 2B Decrease of fluorescence intensity of DiR SBS liposomes in rat lungs after intratracheal administration.

Note: $\mathrm{n}=3$ for each time point

Abbreviations: SBS, salbutamol sulfate; DiR, I,I'-dioctadecyltetramethyl indotricarbocyanine iodide. the respiratory tissues decreased with a first order rate constant of $0.123 \mathrm{~h}^{-1}$.

\section{Pharmacokinetic analysis in rat plasma}

SBS is a typical water-soluble active pharmaceutical ingredient. It has been reported that SBS can be absorbed to its maximum concentration as early as 10 minutes after administration, ${ }^{38}$ suggesting that SBS can be immediately absorbed into the circulation system once it is released from the liposome at the respiratory tract. In order to evaluate the sustained release effect of SBS liposome formulation in the lungs, the pharmacokinetics of SBS were analyzed by collecting serial plasma samples from rats after intratracheal administration of SBS liposome suspension or free SBS solution (Table 3). The mean residence time for the SBS liposome group (18.73 \pm 2.54 hours) was greater than that for the free SBS solution group (8.09 \pm 2.65 hours) $(P<0.05)$, suggesting that entrapped SBS had remained in the pulmonary system longer. Figure 3 further reveals that liposomes significantly slowed the elimination of entrapped SBS from the lungs; the free SBS solution group showed a significantly higher SBS concentration $(1139.78 \pm 180.61 \mathrm{ng} / \mathrm{mL})$ in plasma at 5 minutes when compared to the SBS liposome suspension group $(124.46 \pm 84.64 \mathrm{ng} / \mathrm{mL})$. These data suggest that free or unencapsulated SBS could transport across alveolar membrane immediately but liposomes could prevent the release and transportation of entrapped SBS into the blood. There was no detectable SBS in plasma for the free SBS solution group after 24 hours, whereas an average concentration of $11.62 \pm 5.13 \mathrm{ng} / \mathrm{mL}$ was still observed for the SBS liposome group at 48 hours (Figure 3), therefore implying that liposomal SBS was slowly released from the lungs. The pharmacokinetic findings suggest that liposome formulations could maintain the localization of SBS in pulmonary tissues. The relationship between liposomal SBS remaining in pulmonary tissues and its anti-asthmatic effect was further investigated in the studies below.

Table 3 Pharmacokinetic analysis of SBS in the plasma of rats after pulmonary administration of SBS liposome formulation or free SBS solution

\begin{tabular}{lcc}
\hline Parameters & Liposome SBS & Free SBS solution \\
\hline $\mathrm{AUC}_{0-\infty}(\mathrm{ng} / \mathrm{mL} \times \mathrm{h})$ & $1376.58 \pm 598.22$ & $147 \mathrm{I} \pm 733.88$ \\
$\mathrm{MRT}(\mathrm{h})^{\mathrm{a}}$ & $18.73 \pm 2.54$ & $8.09 \pm 2.65$ \\
$\mathrm{C}_{\max }(\mathrm{ng} / \mathrm{mL})$ & $168.45 \pm 77.94$ & $\mid 139.78 \pm 180.6 \mathrm{I}$ \\
$\mathrm{T}_{\max }(\mathrm{h})$ & $2.38 \pm 0.89$ & $-^{\mathrm{b}}$ \\
\hline
\end{tabular}

Notes: Data were expressed as mean \pm SD $(n=5)$. 'Student's $t$-test: liposome SBS versus free SBS solution, $P<0.05$; there was no $T_{\max }$ for free SBS solution.

Abbreviations: MRT, mean residence time; $\mathrm{AUC}_{0}{ }_{h-\infty}$, area under the curves from time zero to infinity; SBS, salbutamol sulfate; SD, standard deviation. 


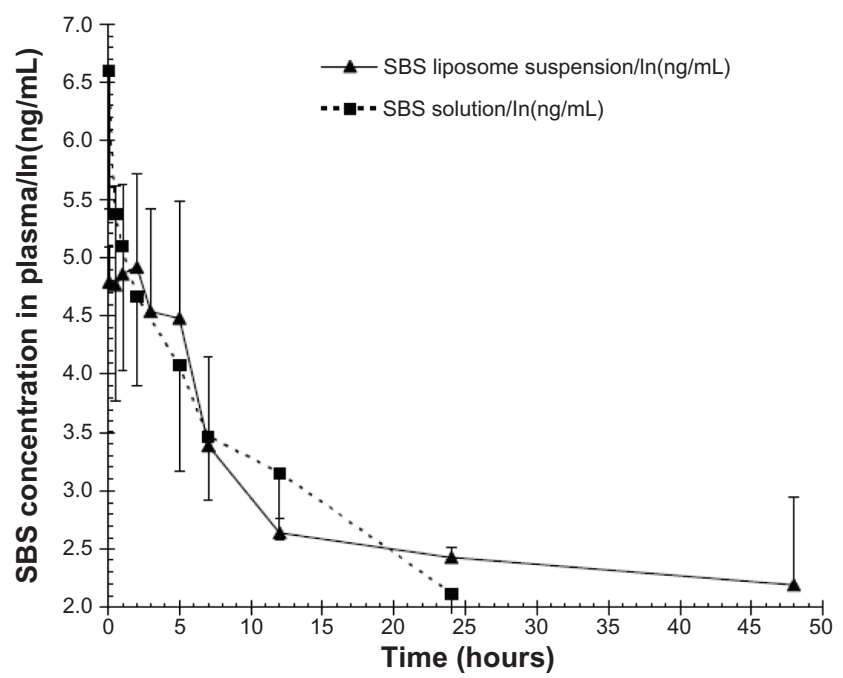

Figure 3 SBS concentration in the plasma of rats at different time points after pulmonary administration of SBS liposome formulation or free SBS solution.

Note: Data were expressed as mean \pm SD $(n=5)$.

Abbreviations: SBS, salbutamol sulfate; SD, standard deviation.

\section{Concentration and disposition of SBS in rat lungs}

The sustained release profile of SBS liposomes was evaluated by measuring the SBS concentrations in the rat lungs at different time points after pulmonary administration of SBS liposomes. Appreciable increases in the mean residence time and area under the curves from time zero to infinity $\left(\mathrm{AUC}_{0 \mathrm{~h}-\infty}\right)$ of SBS remaining in pulmonary tissues were observed in rats administered with SBS liposome suspension (Table 4; $P<0.05)$. Particularly, the mean residence time of the SBS liposome group ( $21.85 \pm 1.41$ hours) was seven-fold longer compared to that of the free SBS solution group (3.27 \pm 0.07 hours), whereas $\mathrm{AUC}_{0 \mathrm{~h}-\infty}$ of the SBS liposome group $(1061.37 \pm 27.20 \mu \mathrm{g} / \mathrm{g} \cdot \mathrm{h})$ was six-fold higher than that of the free SBS solution group $(173.05 \pm 21.36 \mu \mathrm{g} / \mathrm{g} \cdot \mathrm{h})$. As shown in Figure 4, the free SBS solution group showed a significantly lower concentration of SBS in the lungs compared to the SBS liposome group, suggesting that free SBS solution could travel across the alveolar membrane, quickly spreading into the circulation. During the first 12 hours after administration of the SBS liposome suspension, SBS

Table 4 Parameters of the various formulations of SBS in rat lungs after pulmonary administration (mean $\pm S D, n=3)$

\begin{tabular}{lcc}
\hline Parameters & Liposome SBS & Free SBS solution \\
\hline AUC $_{0-\infty}(\mu g / g \times h)^{\mathrm{a}}$ & $1061.37 \pm 27.20^{\mathrm{a}}$ & $173.05 \pm 21.36$ \\
${\text { MRT }(\mathrm{h})^{\mathrm{a}}}$ & $21.85 \pm 1.4 \mathrm{I}^{\mathrm{a}}$ & $3.27 \pm 0.07$ \\
Clearance $^{\mathrm{a}}$ & $6.39 \pm 0.20^{\mathrm{a}}$ & $44.3 \mathrm{I} \pm 4.32$ \\
\hline
\end{tabular}

Notes: aStudent's t-test: liposome SBS versus free SBS solution, $P<0.05$.

Abbreviation: $\mathrm{AUC}_{0 \mathrm{h- \infty}}$, area under the curves from time zero to infinity; MRT, mean residence time; SBS, salbutamol sulfate; SD, standard deviation.

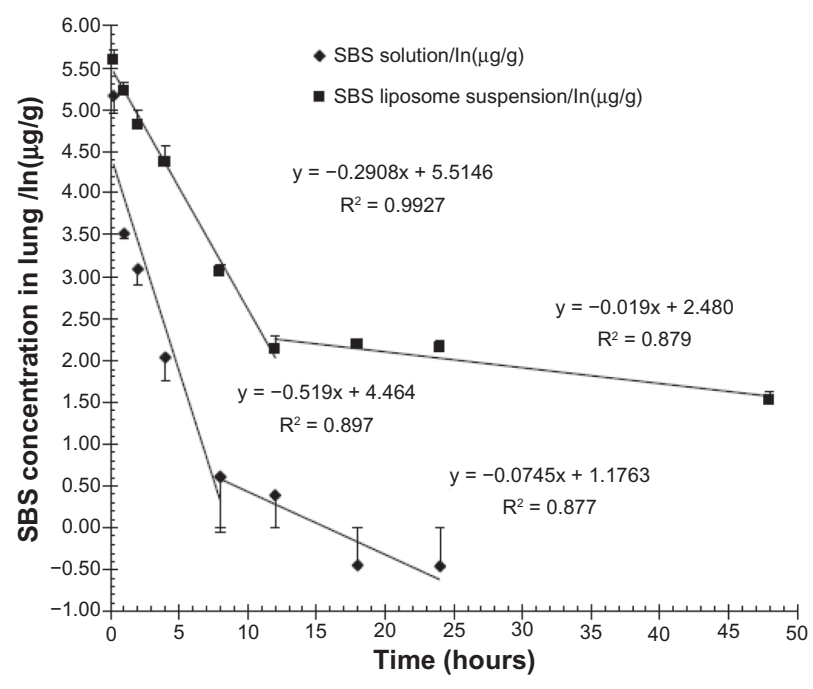

Figure 4 SBS concentration in the lungs of rats at different time points after pulmonary administration of SBS liposome formulation or free SBS solution. Note: Data were expressed as mean $\pm S D(n=3)$.

Abbreviations: SBS, salbutamol sulfate; SD, standard deviation.

concentration in the lungs decreased in a first-order fashion with a rate constant of $0.291 \mathrm{~h}^{-1}$. In contrast, during the first 8 hours after administration of free SBS solution, SBS concentration in the lungs decreased with a first-order rate constant of $0.519 \mathrm{~h}^{-1}$. These data indicated that the migration of SBS from the lungs to the plasma was nearly two-fold slower when it was delivered in liposome suspension. Moreover, the elimination rate for SBS $\left(0.291 \mathrm{~h}^{-1}\right)$ was shown to be much faster than that of $\operatorname{DiR}\left(0.123 \mathrm{~h}^{-1}\right)$, suggesting that pulmonary elimination of lipids may be half as fast as that of SBS. Liposome formulations could prevent the rapid release and elimination of SBS from the pulmonary tissues, resulting in a higher SBS concentration remaining in the lungs. These data implicate the potential use of liposomes for prolonging the anti-asthmatic effect of SBS.

\section{Pharmacodynamic study of guinea pigs}

To investigate the anti-asthmatic effect of SBS liposomes, pharmacodynamic analysis was performed using the Konzett-Rössler method. ${ }^{24}$ Since Sprague Dawley rats are insensitive to our asthma model, guinea pigs were used instead in this part of the study, and those guinea pigs with an asthma latent period exceeding 120 seconds were not included. Three groups of guinea pigs ( $\mathrm{n}=6$ per group) given SBS liposomes, free SBS solution, or saline were observed for anti-asthmatic effect after spraying of histamine. Histamine is known to play an important role in bronchi constriction mediated by histamine receptors causing bronchial asthma attack ${ }^{39}$ and was used to induce asthma in our 
guinea pig model. An anti-asthmatic effect was considered to be present when the animal did not twitch and fall down for at least 15 minutes (asthma latent period) after histamine treatment. The two groups of guinea pigs treated with SBS liposome suspension or free SBS solution displayed the antiasthmatic effect, whereas no anti-asthmatic effects were observed for the saline blank group (Figure 5). The SBS liposome group showed an apparent anti-asthmatic effect for up to 18 hours after pulmonary administration. In contrast, the free SBS solution group maintained the anti-asthmatic effect for only up to 6 hours. At 8 hours after pulmonary administration, the free SBS solution group was found to twitch and fall down as a result of histamine treatment, and the asthma latent period was $134 \pm 27$ seconds (compared to $98.3 \pm 18$ seconds for the saline blank group, $P<0.05$ ), indicating that the anti-asthmatic effect of free SBS solution had ceased by that time point. These pharmacodynamic data were found to correspond with the time-dependent concentrations of liposomal SBS or free SBS observed in rat lungs after pulmonary delivery (Figure 4), showing that the antiasthmatic effect of entrapped SBS was correlated with retention of SBS by liposomes in the lungs. Moreover, our guinea pig model displayed rapid and persistent acting of SBS liposomes for the treatment of acute asthma. Taking all the in vivo data into consideration, it can be concluded that liposome formulation may allow localization of SBS in the pulmonary tissues contributing to sustained release of SBS and thus exerting a prolonged anti-asthmatic effect.

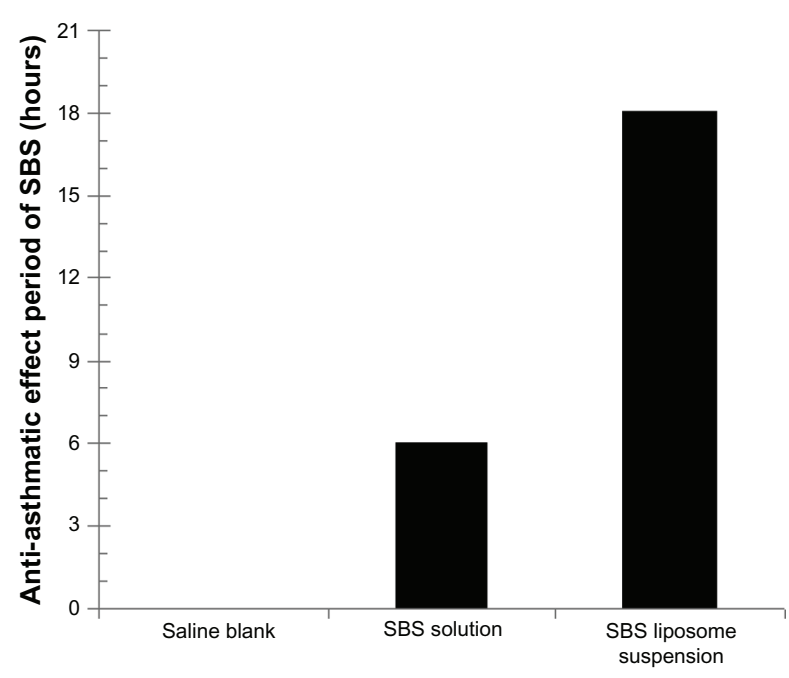

Figure 5 Anti-asthmatic effect periods in guinea pigs given saline, SBS solution, or SBS liposomes ( $n=6$ per group) before spraying of $0.4 \%$ histamine saline solution for 20 seconds at the rate of $70 \mathrm{~L} /$ minute.

Note: Anti-asthmatic effect was considered to be present when the animal did not twitch and fall down for at least 15 minutes (asthma latent period) after histamine treatment.

Abbreviation: SBS, salbutamol sulfate.

\section{Conclusion}

Liposomes showed promising properties as a drug delivery system for SBS. Water-soluble SBS could be efficiently encapsulated into liposomes prepared by the VPG method. The optimal EE was achieved by use of isotonic agents. In addition to the sustained release effect of the formulation, unencapsulated SBS in the liposome suspension may provide an immediate bronchodilating effect. In vivo animal studies showed that SBS was released from liposomes to act on the $\beta_{2}$ receptor in the pulmonary tissues leading to the bronchodilating effect. Fluorescence images of DiR SBS liposomes indicated that liposomes were homogeneously distributed in the lungs and the total decrease of radiation intensity suggested that lipids and DiR itself may be eliminated in time from the respiratory tissues. The pharmacodynamic study of guinea pigs demonstrated a significantly longer anti-asthmatic effect by SBS liposome suspension compared to free SBS solution. In summary, the present study suggests higher therapeutic efficacy for SBS by liposome-mediated pulmonary delivery, as this approach can reduce the frequency of administration by increasing the drug retention time in the lungs and avoid systemic side effects due to accumulating drug after frequent administration. Future studies may focus on the modification of liposomes by using polyethylene glycol to enhance the stability of liposomes in vivo and, furthermore, by conjugating with targeting ligands to enrich site-specific therapeutic action.

\section{Acknowledgments}

We would like to thank Mr Mulin Yang, Leslie Dan Faculty of Pharmacy, University of Toronto, ON, Canada, for proofreading the article; and thank Hong Kong Baptist University for the Faculty Research Grant (FRG2/08-09/089) support.

\section{Disclosure}

The authors report no conflicts of interest in this work.

\section{References}

1. Eagan TM, Brøgger JC, Eide GE, Bakke PS. The incidence of adult asthma: a review. Int J Tuberc Lung Dis. 2005;9(6):603-612.

2. Bryan J. Ventolin remains a breath of fresh air for asthma sufferers, after 40 years. Pharm J. 2007;279(7473):404-405.

3. Kelly HW, Murphy S. Beta-adrenergic agonists for acute, severe asthma. Ann Pharmacother. 1992;26(1):81-91.

4. Liisa ML, Kirsi P, Kaarina K, Seija Tammilehto. Decomposition of salbutamol in aqueous solutions. II. The effect of buffer species, $\mathrm{pH}$, buffer concentration and antioxidants. Int J Pharm. 1995;117(2): 189-195

5. Nizet TA, Broeders ME, Folgering HT. Tremor side effects of salbutamol, quantified by a laser pointer technique. Respir Med. 2004;98(9): 844-850. 
6. Bendas ER, Tadros MI. Enhanced transdermal delivery of salbutamol sulfate via ethosomes. AAPS Pharm Sci Tech. 2007;8(4):E07.

7. Sweetman SC. Martindale: The Complete Drug Reference. London, UK: Pharmaceutical Press; 2005.

8. Liu FY, Shao Z, Kildsig DO, Mitra AK. Pulmonary delivery of free and liposome insulin. Pharm Res. 1993;10(2):228-232.

9. Beyer J, Barzen G, Risse G, et al. Aerosol amphotericin B for prevention of invasive pulmonary aspergillosis. Antimicrob Agents Chemother. 1993;37(6):1367-1371.

10. Gavalda J, Martin MT, Lopez P, et al. Efficacy of nebulized liposome amphotericin B in treatment of experimental pulmonary aspergillosis. Antimicrob Agents Chemother. 2005;49(7):3028-3030.

11. Okumura K, Iwakawa S, Yoshida T, Seki T, Komada F. Intratracheal delivery of insulin and adsorption from solution and aerosol by rat lung. Int J Pharm. 1992;88(1-3):63-73.

12. Yamamoto A, Umemori S, Muranishi S. Absorption enhancement of intrapulmonary administered insulin by various absorption enhancers and protease inhibitors in rat. $J$ Pharm Pharmacol. 1994;46(1):14-18.

13. Poyner EA, Alpar HO, Almeida AJ, et al. A comparative study on the pulmonary delivery of tobramycin encapsulated into liposomes and PLA microspheres following intravenous and endotracheal delivery. J Control Release. 1995;35(1):41-48.

14. Ten RM, Anderson PM, Zein NN, Temesgen Z, Clawson ML, Weiss W. Interleukin-2 liposomes for primary immune deficiency using the aerosol route. Int Immunopharmacol. 2002;2(2-3):333-344.

15. Edwards D, Hanes J, Caponetti G, et al. Large porous aerosols for pulmonary drug delivery. Science. 1997;276(5320):1868-1871.

16. Saari M, Vidgren MT, Koskinen MO, Turjanmaa VM, Nieminen MM. Pulmonary distribution and clearance of two beclomethasone liposome formulations in healthy volunteers. Int J Pharm. 1999;181(1):1-9.

17. Huang YY, Wang CH. Pulmonary delivery of insulin by liposomes. J Control Release. 2006;113(1):9-14.

18. Brandl M, Massing U. Vesicular phospholipid ges. In: Torchilin VP, Weissig V, editors. Liposomes. 2nd ed. New York: Oxford Press; 2003: 353-372.

19. Yang ZJ, Fong DWE, Yin LL, Wong Y, Huang W. Liposomes modulate docetaxel-induced lipod oxidization and membrane damage in human hepatoma cells. J Liposome Res. 2009;19(2):122-130.

20. Zhigaltsec IV, Maurer N, Edwards K, Karlsson G, Cullis PR. Formation of drug-arylsulfonate complexes inside liposomes: A novel approach to improve drug retention. J Control Rel. 2006;110(2):378-386.

21. Wall DA, Pierdomenico D, Wilson G. An in vitro pulmonary epithelial system for evaluating peptide transport. J Control Rel. 1993;24(1-3): $227-235$.

22. Okumura S, Fukuda Y, Takahashi K, Fujita T, Yamamoto A, Muranishi S. Transport of drugs across the Xenopus pulmonary membrane and their absorption enhancement by various absorption enhancers. Pharm Res. 1996;13(8):1247-1251.

23. Liu Y, Lu WL, Hu X, et al. Transport of Recombinant hirudin across Xenopus pulmonary membrane. Chin Pharm J. 2005;39(17): $1318-1322$.
24. Vogel HG. Bronchospasmolytic activity in anesthetized guinea pigs. In: Vogel H, editor. Drug Discovery and Evaluation: Pharmacological Assays. 2nd ed. New York: Springer; 2002:359-360.

25. Nogami-Itoh M, Yakuo I, Hammerbeck DM, Miller RL, Takeyama K. The equivalent bronchodilator effects of salbutamol formulated in chlorofluorocarbon and hydrofluoroalkane-134a metered dose inhalers on the histamine-induced pulmonary response in dogs. Pharm Res. 1997;14(2): 208-212.

26. Venables KM, Dally MB, Burge PS, Pickering CA, Nerman Taylor AJ. Occupational asthma in a steel coating plant. $B J$ Ind Med. 1985; 42(8):517-524.

27. Brandl M, Achmann BD, Drechsler M, Bauer KH. Liposome preparation by a new high pressure homogenizer Gaulin Micron Lab 40. Drug Dev Ind Pharm. 1990;16(14):2167-2191.

28. Kaiser N, Kimpfler A, Massing U, et al. 5-Fluoroacil in vesicular phospholipid gels for anticancer treatment: entrapment and release properties. Int J Pharm. 2003;256(1-):123-131.

29. Brandl M, Drechsler M, Bachmann D, Tardi C, Schmidtgen M, Bauer KH. Preparation and characterisation of semisolid phospholipid dispersions and dilutions thereof. Int J Pharm. 1998;170(2):187-199.

30. Brandl M, Reszka R. Preparation and characterisation of phospholipid membrane gels as depot formulations for potential use as implants. Proc Int Symp Control Release Bioact Mater. 1995;22:472-473.

31. Brandl M, Drechsler M, Bachmann D, Bauer KH. Morphology of semisolid aqueous phosphatidylcholine dispersions, a freeze fracture electron microscopy study. Chem Phys Lipids. 1997;87(1):65-72.

32. Brandl M, Tardi C, Drechsler M, et al. Three-dimensional liposome networks: freeze fracture electron microscopical evaluation of their structure and in-vitro analysis of release of hydrophilic markers. $A d v$ Drug Deliv Rev. 1997;24(2-3):161-164.

33. Kalchenko V, Shivtiel S, Malina V, et al. Use of lipophilic near-infrared dye in whole-body optical imaging of hematopoietic cell homing. J Biomed Opt. 2006;11(5):050507.

34. Vladimir T, Volkmar W. Liposomes: A Practical Approach. 2nd ed. New York: Oxford University Press; 2003.

35. Juliano RL, McCullough HN. Controlled delivery of an antitumor drug: localized action of liposome encapsulated cytosine arabinoside administered via the respiratory system. J Pharmacol Exp Ther. 1980;214(2): 381-387.

36. Taylor KM, Taylor G, Kellaway IW, Stevens J. The influence of liposomal encapsulation on sodium cromoglycate pharmacokinetics in man. Pharm Res. 1989;6(7):633-636.

37. Rao J, Dragulescu-Andrasi A, Yao H. Fluorescence imaging in vivo: recent advances. Curr Opin Biotechnol. 2007;181(1):17-25.

38. Lipworth BJ, Clark DJ. Lung delivery of salbutamol by dry powder inhaler (Turbuhaler ${ }^{\mathbb{B}}$ ) and small volume antistatic metal spacer (Airomir $^{\circledR}$ CFC-free MDI plus NebuChamber ${ }^{\circledR}$ ). Eur Respir J. 1997;10(8):1820-1823.

39. Yuji T, Hirokazu K, Ryuta H, et al. Roles of histamine receptor in a guinea pigs asthma model. Int J Immunopharmacol. 1998;20(10): $565-571$.
International Journal of Nanomedicine

\section{Publish your work in this journal}

The International Journal of Nanomedicine is an international, peerreviewed journal focusing on the application of nanotechnology in diagnostics, therapeutics, and drug delivery systems throughout the biomedical field. This journal is indexed on PubMed Central, MedLine, CAS, SciSearch ${ }^{\circledR}$, Current Contents ${ }^{\circledR} /$ Clinical Medicine, Journal Submit your manuscript here: http://www.dovepress.com/international-journal-of-nanomedicine-journa

\section{Dovepress}

Citation Reports/Science Edition, EMBase, Scopus and the Elsevier Bibliographic databases. The manuscript management system is completely online and includes a very quick and fair peer-review system, which is all easy to use. Visit http://www.dovepress.com/ testimonials.php to read real quotes from published authors. 